\title{
Role of Atomic Migration in Nanocrystalline Stability: Grain Size and Thin Film Stress States
}

\author{
Monica Kapoor and Gregory B. Thompson* \\ The University of Alabama \\ Department of Metallurgical \& Materials Engineering \\ Tuscaloosa, AL USA 35487-0202 \\ *Gthompson@eng.ua.edu
}

\begin{abstract}
As the length scale of materials decreases to the nanometer regime, grain boundaries occupy a relatively larger volume fraction. Consequently, they play an important role in stabilizing nanocrystalline systems. This review looks at the role of solute segregation to grain boundaries in stabilizing such systems. In recent years, grain size stabilization from solute segregation has led to new types of thermodynamic stability maps as a materials design tool. We propose to extend and adapt these concepts of grain boundary solute segregation as a stabilizing effect to thin film stress states. A recent study on Fe-Pt alloy films, where one species enriched the boundaries, was shown to manipulate the stress from tensile-to-compressive as a function of composition. This suggests that intrinsic segregation can be used as a tunable variable to manipulate stress states, analogous to changing film processing parameters, such as deposition rate, pressure, etc. The application of such solute segregation is at the precipice of new opportunities in materials design of thin films.
\end{abstract}




\section{Introduction}

When the grain size of a material is reduced to nanometer regime, interfaces constitute a large part of the overall structure [1]. This is aptly illustrated in Fig. 1, which shows how the fraction of intercrystalline regions (the lattice volume near the grain boundaries which differs from the equilibrium structure), the grain boundaries, and the triple junctions (where three grains/crystals intersect) varies as a function of the grain size. When the grain size decreases from $100 \mathrm{~nm}$ to $3 \mathrm{~nm}$, the grain boundary fraction increases from $3 \%$ to $44 \%$, or, alternatively, a larger fraction of the atoms is at the interfaces. Consequently, nanocrystalline materials can and often do exhibit different properties - such as crystallographic structure [2], solubility [3] or mechanical properties [4] - than their bulk forms.

These nanocrystalline materials are processed in a variety of methods, including physical or chemical vapor deposition and electro-deposition [5] as thin films as well as ball-milling and sintering of powders into monolithic forms [6]. In each case, the stability of the nanocrystalline structure is necessary for the processed material to maintain a desired set of properties or attributes. Understanding this nanocrystalline stability has been an area of active research for several decades [5, 7-9]. Most recently, several researchers across different disciplines have been addressing how atomic segregation to grain boundaries contributes to nanocrystalline stability.

The motivation of this review is to understand how such atomic migration and segregation to nanocrystalline grain boundaries evolves and contributes to 
the stability in thin films and nanocrystalline alloy materials. In particular, thin films and coatings are sensitive to delamination and cracking, which is a product of the residual stress [5]. Recent work in nanocrystalline alloys, in both thin film and monolithic forms, has shown that segregation to grain boundaries can have a stabilizing effect $[10,11]$. These studies have primarily addressed stability against grain growth. Interestingly, the role of segregation has largely been overlooked as a means to stabilize the stress state in nanocrystalline grains in post-coalesced thin films. In this review, the current status of atomic migration and segregation as a stabilizing effect on grain size and thin film stress states is addressed.

First, we will discuss how solute segregation to grain boundaries stabilizes grain size in nanocrystalline systems. The high surface area-to-volume ratio in such structures leads to a larger fraction of non-equilibrium grain boundary sites, altering the 'bulk' thermodynamics. A number of experiments on stabilizing nanocrystalline alloys, ranging from strongly segregating systems such as Ni-P to weakly segregating systems such as $\mathrm{Ni}-\mathrm{W}$, have provided new insights into the factors that contribute to stabilization $[8,10]$. These efforts in conjunction with computational modeling have very recently culminated in thermodynamic stability maps which appear to be a promising design tool [12-16].

Second, we will discuss the role of grain boundaries in the stress generation in a growing thin film. The generation of intrinsic or residual stress in thin films has been debated upon for several years with recent experiments and 
simulations providing evidence to support the importance of grain boundaries in the stress evolution [17-27].

In both topics, the role of atomic migration into and out of a nanocrystalline boundary is paramount in the stability of the structure.

\section{Segregation-induced nanocrystalline stability}

In a polycrystalline system, the free energy will decrease by reducing the grain boundary area. This results in an inherent driving force to reduce the grain boundary area by grain growth and thereby creating instability with respect to the grain size. Two primary approaches have been used to stabilize the nanocrystalline alloys $[6,9,28]-(1)$ The kinetic approach, which is pinning the boundaries to reduce their mobility. This pinning is accomplished by forming precipitates (Zener pinning), solute drag, and/or chemical ordering [29, 30]. Unfortunately, these approaches are overcome at high temperatures and the

grain stability is lost $[6,11]$. (2) The thermodynamic approach, which is stabilizing the nanocrystalline alloy by reducing the driving force for growth. This can be achieved by allowing a solute to segregate to the grain boundary and altering the thermodynamic energy balance [31-33]. This latter concept is the primary focus below.

1. Thermodynamics of grain size stabilization

Some 20 years ago, the concept of solute segregation to the grain boundaries to stabilize nanocrystalline alloys was proposed and eventually 
developed by other researchers $[3,7,8,10-14,16,31-38]$. One of the earliest attempts to study and quantify the effect of solute segregation was in Ni-P system $[8,35]$. In a Ni-3.6 at. \%P alloy, grain size increased from $\sim 9 \mathrm{~nm}$ to $\sim 25$ $\mathrm{nm}$ after annealing at $400^{\circ} \mathrm{C}$. The limited grain gro wth was attributed to $\mathrm{P}$ segregation [35] with atom probe tomography revealing that $P$ was inhomogenously distributed at the grain boundaries. Using a mass balance for the solute, Kirchheim group derived an equation which relates the solute content to the grain size by the empirical equation -

$$
d\left(c_{0}\right)=\frac{\Delta}{1-\left(\frac{c_{0}-c g b}{c_{g}-c_{g b}}\right)^{\frac{1}{3}}}
$$

where $d=$ grain size, $\Delta=$ grain boundary width, $c_{0}=$ bulk solute concentration, $c_{g}=$ solute concentration in the grain interior, and $c_{g b}=$ solute concentration at the grain boundary. This equation predicted that the stable grain size would decrease as a function of increasing $\mathrm{P}$ content. This relationship, with subsequent modifications by several other groups, has been shown to predict the stable grain sizes in Ni-P alloys as well as being consistent with the newer observations in Ni-W and Fe-Zr systems [7, 39].

This stabilization is explained by thermodynamics as follows [31, 33, 36]: The change in Gibbs free energy, $G$, of a polycrystalline material due to the change in grain boundary area, $a$, is given by -

$$
d G=\gamma d a
$$

where $\gamma=$ grain boundary energy. In single-component systems the grain boundary energy, $\gamma$, is positive, so the only way to lower the free energy is by reducing the grain boundary area, hence an inherent driving force for grain 
growth. However, if the grain boundary energy can somehow be reduced to zero, then there is no driving force and the system will be stable. This reduction in grain boundary energy can be accomplished in an alloy system by allowing a solute to segregate to the grain boundaries and alter $\gamma[31,33,36]$. The grain boundary energy due to segregation is then given as -

$$
\gamma=\gamma_{0}-\Gamma(\Delta G \operatorname{seg}+R T \ln X)
$$

where $\gamma_{0}=$ grain boundary energy of the pure element, $\Gamma=$ specific excess of solute atoms located at the grain boundary area, $\Delta G$ seg $=$ segregation energy, $R$ $=$ gas constant, $T=$ temperature and $X=$ global composition. This equation shows that grain boundary energy of a pure element, $\gamma_{0}$, can be reduced by the energy needed to move the solute atoms to the grain boundary. With a sufficiently high segregation energy, the solute excess can actually reduce the grain boundary energy, $\gamma$, to zero thereby eliminating the driving force for grain growth.

\section{Factors affecting stabilization}

Equation (3) has been fitted to Fe-P, Ni-P, and Pd-Zr experimental data and showed good agreement [32]. An outcome of that work demonstrated that as the solute content increased, the grain boundary reached a saturation value with respect to solute atoms it can accommodate. At this saturation point, a stable grain size was achieved $[32,36]$. From the results of these particular 
systems, it was posited that solute segregation in a strongly segregating system will drive the grain boundary energy to zero [32].

More recently, Detor and Schuh reported similar grain size stabilization in Ni-W alloy, which is a weakly segregating system [7]. In such a system, the grain boundary energy would not necessarily have to be zero to achieve stabilization in contrast to prior rationalization for strongly segregating systems. Simulations on Ni-W alloys with 1, 5 and 20 at. \% W showed that the tendency of a solute to segregate decreased as the solute concentration increased [10]. For a grain size of $4 \mathrm{~nm}$, the enrichment factor (i.e. ratio of solute concentration at the grain boundary to that in the bulk) decreased from $\sim 2.6$ to $\sim 1$ as the bulk solute composition increased from 1 at. $\%$ to 20 at. \%. This interestingly translated to enthalpy of segregation decreasing as a function of increasing bulk composition, as seen in Fig. 2 [10]. This composition-dependent segregation enthalpy had not been previously considered. This composition-dependent segregation enthalpy was now used to calculate the change in grain boundary energy in Ni-P and in $\mathrm{Ni}-\mathrm{W}$. They found that grain boundary energy does indeed go to zero in the Ni-P system but it was still positive in the Ni-W system. Thus, it is insufficient to only consider high enthalpy of segregation as the deciding factor for nanocrystalline stabilization. To rationalize this stabilization, other factors must be considered.

It has been proposed that not only energy of segregation but its relation to the energy of mixing determines nanocrystalline stability $[14,15]$. For example, there are many systems with high energies of segregation, but at high solute concentrations or high temperatures these solutes agglomerate and form a 
phase separated microstructure which limits their use in nanocrystalline stabilization [11]. In contrast, there are many alloys with low segregation enthalpies which are successful nanocrystalline alloys, e.g. Ni-W [7]. The competition between these two energies must be considered. By plotting the alloys as a function of the enthalpy of mixing and enthalpy of segregation, a thermodynamic stability map for nanocrystalline alloys can be created [14-16]. These maps can be used as a tool to quickly screen a variety of alloys for nanocrystalline stability. Two general mapping schemes have been proposed and we review both of them below $[14,15]$.

\section{Thermodynamic Stability Maps}

Schuh's group used a modified regular nanocrystalline solution (RNS) model and included stability against phase separation as an input to develop the thermodynamic stability maps $[12,15]$. The RNS model allows for distinct grain boundary and grain interior regions to be modeled as a system [38]. The RNS model was adapted to calculate segregation enthalpies (independent of grain boundary solute and temperature), which is not an easily available material property and is usually estimated. In their work they have considered $\sim 2500$ binary alloys and mapped these alloys as a function of enthalpy of mixing and enthalpy of segregation. Fig. 3 is one such thermodynamic stability map with pink and green regions with a sliver region between the two in yellow. Each of these regions indicate different levels of stability [15]. The solute and the solvent are denoted by a combination of the element abbreviation and geometrical 
symbols respectively. An alloy in the pink region is not stable as a nanocrystalline system, in the yellow region is stable as a nanocrystalline system but not against phase separation, and in the green region is stable as a nanocrystalline system and against phase separation.

To understand these maps, we will go through the highlights of how such a thermodynamic stability map is obtained - (1) A free energy surface, shown in Fig. 3 (b), is computed in the grain boundary concentration and grain size space. If there is no minimum in this surface, then it cannot be stabilized as a nanocrystalline system, and will be plotted in the pink region of the thermodynamic stability map. For example, Mn solute in the $\mathrm{Cu}$ solvent is the $\mathrm{Mn}$ abbreviation next to the filled blue circle in the pink region (labeled by the solid arrow) indicates that $\mathrm{Mn}$ solute in $\mathrm{Cu}$ cannot be stabilized as a nanocrystalline system. (2) If there is a minimum in the surface, then it will be stabilized as a nanocrystalline system, at the particular grain size and grain boundary composition. Fig. 3 (b) shows a surface with the minimum, marked by an arrow. If the minimum energy of this surface is lower than that of other possible bulk states, then this system is stable as a nanocrystalline system and against phase separation and will be plotted in the green region in the map. For example, Au with the open magenta circle in the green region indicates that $\mathrm{Au}$ solute in a Mo solvent will be a stable nanocrystalline system. (3) If the minimum in the surface is not lower than other possible bulk states, then this system will be stable as a nanocrystalline system but not against phase separation. 
These types of maps have led to some unexpected predictions. For example, consider the solute additions to W. In general, nanostructural stability has been estimated based on atomic size mismatch or solubility, which are indication of enthalpy of segregation. However, $\mathrm{Sr}$ in $\mathrm{W}$ has the highest atomic mismatch and $\mathrm{Ag}$ and $\mathrm{Cu}$ in $\mathrm{W}$ have the lowest solubility; but all three of these solutes are predicted to be unstable as nanocrystalline systems in reference [16]. Hence, these prior simple 'rules' do not necessarily provide the correct predictions.

Koch's group have modified the above RNS approach to develop a model specifically for strongly segregating systems [40]. They noted that Schuh group's model did not suitably scale the enthalpy of segregation by the grain boundary volume fraction. To date, Koch's group's modification has been applied to only a few systems (Fe-Zr, Cu-Zr, Cu-Nb, and $\mathrm{Ni}-\mathrm{W})$ with some experimental validation of those results [11].

Another set of thermodynamic stability maps has been developed by Darling et al. [14]. In their model, they assume no secondary phase precipitation, which is reasonable in a dilute limit. They modified the Wynblatt-Ku model for surface segregation by replacing the surface environment with a grain boundary interface. Darling et al. suggested that the elastic misfit between the solute and solvent induces solute segregation $[28,41]$. Motivated by prior work on $\mathrm{Pd}-\mathrm{Zr}$ and their work on Fe-Zr alloys, they used elastic size misfit energies as an indication of energy of segregation $[13,39]$. To understand the utility of these maps, we will briefly consider the Fe-Zr alloy. 
Fig. 4 (a) shows the normalized grain boundary energy as a function of the solute $\mathrm{Zr}$ in Fe for a series of grain sizes, with the calculation performed for 3 at. $\% \mathrm{Zr}$ at $550^{\circ} \mathrm{C}$. It can be seen that a grain size of $23.1 \mathrm{~nm}$ gives a zero grain boundary energy and will be the stable at $550^{\circ} \mathrm{C}$. These modeling predictions are consistent with experimental grain size and calculated enthalpy of mixing for Fe- 4 at. \% Zr [14]. Fig. 4(a) clearly indicates that the bulk solute concentration for stabilization will vary with grain size.

Taking this concept (obtaining zero grain boundary energy) and applying it to a series of solutes led to the conclusion that stable solutes are a function of elastic enthalpy and enthalpy of mixing. Plotting the solutes as a function of both resulted in stability maps of solutes for a given solvent. Fig. 4(b) is such a map of the Fe solvent - with several different solutes - that will stabilize a grain size of $25 \mathrm{~nm}[14,42]$. The red dots represent the favorable or stabilizing solutes, with the size of the dot corresponding to the minimum amount of solute required to stabilize a particular targeted grain size, i.e. $25 \mathrm{~nm}$ in this case.

All of these maps fit the limited experimental data very well giving validity that both the enthalpy of mixing and segregation are critical material properties to determine stability. Though each of these groups approached the issue from a slightly different perspective, i.e. calculating enthalpy of segregation (Shuch) compared to using elastic enthalpy as an indication of enthalpy of segregation (Darling et al.).

More recently, Schuh group's has used Monte Carlo analysis to understand the experimental results on ball-milled nanocrystalline $\mathrm{W}-20$ at.\% Ti 
alloy. This alloy had $\sim 20 \mathrm{~nm}$ grain sizes after annealing at $1100^{\circ} \mathrm{C}$ for a week as compared to $\sim 600 \mathrm{~nm}$ grain size for an unalloyed $\mathrm{W}$ system. They found wide regions of solute depletion and enrichment with a periodicity consistent with the grain size and attributed the thermal stability to these regions of solute enrichment which they called 'grain boundary states'. These results indicate that there are more mechanisms and factors contributing to thermal stability than our current understanding $[3,43]$. Clearly more rigorous experimentation is still needed to test the limits of these models.

In the next section, we will discuss how this concept of solute segregation to the grain boundaries can be adapted to stabilize and regulate the stress states in a nanocrystalline thin film.

\section{Stress evolution in thin films}

In the prior section, we established how the role of solutes in grain boundaries is critical in the stabilization of the grain size. When a thin film is deposited, and is not subjected to single crystal epitaxial growth conditions, a polycrystalline structure forms in the film. These grains are typically nanocrystalline and develop into a columnar morphology as the film thickens. The formation of these grains and their boundaries is a result of the nucleation and growth of isolated clusters which eventually impinge into each other creating these boundaries [5]. It has been found that the grain size correlates with the magnitude of the stress state in the thin film $[5,17,19,44]$. Hence the stabilization of grain size is crucial to the stress evolution of a film. 
Understanding stress states of thin films is important because it plays a critical role in the mechanical, electrical, magnetic, and other relevant properties of thin films [45-49]. For example, intrinsic stress states can promote microcracking within the film itself resulting in a loss of connectivity. This would expedite the loss in performance from a micro-electrical connection, microelectromechanical system-based (MEMS) sensors or films used in optical reflection. In extreme cases, films can delaminate from the substrate itself and physically 'roll-up' to relieve its own intrinsic stress state, as seen in Fig. 5 (a) [5, $50,51]$. Clearly, the ability to regulate stress will be able to reduce such failures and possibly enhance the performance properties of thin-film-based technologies and devices.

In general, the stress state has then been controlled by regulating the grain size via processing, i.e. changing the deposition rate, substrate conditions, vacuum pressure, etc. $[19,22,44,52]$. In this section of this review, an analogous link to section 2 is given where solute segregation can be now be used as another means of thin film stress state control. In particular, the stability maps can provide guides to thin film systems where stress states can be manipulated through intrinsic segregation tendencies. We do recognize that film growth is a dynamic process which can place materials far from equilibrium and specific thermodynamic factors influencing each process could differ. Regardless of these challenges, there is evidence showing that solute segregation, very similar to what is seen in nanocrystalline stabilization, can control film stress states. This provides tantalizing possibilities in using the developing 
nanocrystalline stability maps as guides for designing alloy systems where the nanocrystalline grains and stress can be controlled.

\section{Primer on film growth and stress evolution}

As a thin film grows, three basic modes have been identified: (1) island or Volmer-Weber (2) layer or Frank-van der Merwe and (3) a combination of (1) and (2) termed Stranski-Krastanov [5]. In the Volmer-Weber mode, the island formation occurs because the atoms are more strongly bonded to each other than the substrate. As a result, the film exhibits an initial compressive stress as the atomic-scale migration of these atoms form embryonic islands that minimize surface area-to-volumetric energies because of surface capillary effects [53]. Fig. 5 (b) is a schematic of this early stage of growth with the slope of the curve indicating compression (negative) or tension (positive). The subsequent tensile stress originates from the elastic strain associated with the coalescence of these islands to minimize the grain boundary energy [54-56]. Films with low atomic mobility, such as Fe [44, 57], $\mathrm{Cr}$ [57], and $\mathrm{Al}$ [58], tend to retain the tensile condition with continued growth unless acted upon by external stimuli, such as thermal energy. For films with higher intrinsic mobility, the films can exhibit a return to compressive stress which can be partially reduced upon ceasing deposition. If the growth is interrupted at this compressive stress stage, the stress can relax [21, 44, 59]. Upon re-deposition, the compressive stress returns back to the nominal compressive stress measured during growth, hence being a reversible behavior. The mechanisms of post-coalescence compressive stress and stress relaxation are still under discussion. 
Friesen and Thompson [59] proposed that the post-coalescence compressive stress is because of a locked adatom-surface interaction at the substrate. This concept of adatoms trapped on surface ledges in contributing to the stress generation mechanism appears insufficient in explaining recent experimental results by Shin et al. [21]. Pao et al. reported the use of molecular dynamics simulations to study the influence of such defect densities, i.e. atomic surface steps and adatoms, on the film stress evolution [24]. From these results, it was concluded that the magnitude of stress due to such defect densities was inadequate to be the dominant mechanism for the observed compressive stress generation [24].

In other work, Koch et al. [44] observed that during film growth interruption, the value of the relaxation tensile component increased with thin film thickness and grain size. Thus, they proposed that recrystallization and grain growth played an important role in the latter stages of compressive stress evolution and relaxation. However, as pointed by Thompson et al. [60] and Spaepen et al. [61], the recrystallization process is not reversible and this mechanism failed to explain the reversible stress evolution during thin film growth interruption $[20,59]$.

As with Koch et al.'s observation, others have also reported experimental evidence that has shown a correlation in the magnitude of film stress with the grain size (or, inversely, the boundary area) $[17,19,21,23,62]$. To elaborate on this point, Thompson's group used Au films had a median in-plane grain size of 33, 56 and $94 \mathrm{~nm}$ and these were found to have tensile stress relaxation of 80 , 
46 and $29 \mathrm{MPa}$ respectively [17]. In comparison, the control film, which was an epitaxial film with "infinite" grain size, showed a stress relaxation of less than 1 MPa. Further experimental work in other systems has also shown to exhibit this grain size and stress correlation. Flototto et al. reported Ag films deposited on two different substrates, with in-plane grain sizes of $43 \mathrm{~nm}$ and $19 \mathrm{~nm}$, which showed a reversible stress change of 13 and $50 \mathrm{MPa}$ respectively [19]. Abadias et al. reported that the finer the grain size in Mo films resulted in higher tensile stress recovery during coalescence [62]. From these collective experimental results, the grain boundary area appears to directly correlate to stress and the stress relaxation behavior.

To rationalize these results, an explanation by Chason et al. [20] has been proposed that the migration of atoms into and out-of the grain boundaries creates the compressive and relaxation stress recovery, which is developed in detail in the section below.

\section{Adatom migration to grain boundaries}

We will discuss the highlight of Chason's model with a typical stressthickness product as a function of time plot, shown in Fig. 6. This plot of Cu films was obtained in the author's work and shows cycles of deposition/film growth (negative slope), interruption/when the deposition is ceased (positive slope), and resuming film growth (negative slope). For clarity, one representative region each is labeled in Fig. 6. For high mobility atoms, Chason et al. hypothesized that the chemical potential on the surface is higher during deposition which provides a thermodynamic driving force for excess atom migration to the grain boundaries 
[20]. This causes a compressive stress in the grain boundary till the grain boundary saturates and a steady-state is achieved. This region of compression with negative slope is marked with a solid arrow in Fig. 6. When the growth was interrupted (deposition ceased), marked by a dashed arrow in Fig. 6, the chemical potential was lowered on the surface and 'excess' adatoms that diffused to the boundaries now flow out of the boundary to the surface and the compressive stress is relaxed (positive slope). When growth is resumed, the compressive stress returns back to the prior value (negative slope). This return to the former stress state is explained by the change in the chemical potential created from the deposition, which provides the driving force for the atoms back into the grain boundaries generating the renewed compressive stress.

Hence, the driving force for the flux of atoms is chemical potential difference between the surface and the grain boundary given by -

$$
\Delta \mu=\mu_{\mathrm{s}}-\mu_{\mathrm{gb}}=\Delta \mu_{0}+\delta \mu_{\mathrm{s}}+\sigma \Omega
$$

where $\Delta \mu_{0}=$ difference between the surface and grain boundary chemical potential in absence of growth (usually negligible), $\delta \mu_{s}=$ increase in surface chemical potential because of the flux of deposited atoms and accounts for growth conditions, $\sigma=$ stress across the grain boundary, and $\Omega=$ atomic volume. This chemical potential difference is a balance of growth conditions and the stress in the film. This diffusion-based model predicts that the steady state stress in the film is a function of the tensile stress due to the formation of new grain boundaries and its stress relaxation is due to the atoms diffusing into grain boundaries or trapped in atomic ledges given by - 


$$
\sigma_{S S}=\frac{\sigma_{i} R-\sigma_{0}\left[\frac{\left(\Delta \mu_{0}+\delta \mu_{S}\right)}{k T}\right]}{R+\left[\frac{\left(\sigma_{0} \Omega\right)}{k T}\right]}
$$

where $\sigma_{i}=$ tensile stress that develops as a result of the islands coalescing to form grain boundaries, $\mathrm{R}=$ normalized growth rate, $\sigma_{0}=$ compressive stress due to adding a single atom to the grain boundary, $\Omega=$ atomic volume, $\mathrm{T}=$ temperature.

If the steady-state stress is caused by atoms diffusing via grain boundaries, then the time taken to relax this stress (the steady-state time constant) should be proportional to the thickness of the film and the boundary area. In an experimental study with $S n$, a very highly mobility species, the films relaxed completely and confirmed this hypothesis that grain boundary diffusion is a dominate mechanism in stress relaxation [21].

Chason et al. have recently extended this chemical potential based model to include both high and low mobility species [22]. In this modified model, the residual stress is now a function of a dimensionless parameter (D/LR) where $D$ is an effective diffusivity related to the rate of jumping from the surface to the triple junction (grain boundary intersecting with the surface), $R$ is the growth rate, and $L$ is the grain size [22]. This dimensionless parameter reflects a balance of the kinetic processes, such as film growth, grain size and diffusion.

\section{Adatom migration in alloy thin films}

The prior sections have reviewed and established that grain size, hence grain boundary area, are dominant microstructural features in regulating the stress generation in thin films. Much of the prior experimental work has been in 
the use of single species films. In the case of an alloy, with atoms having different diffusivities and segregation energies, mix mobility of multi-component films can offer a tunable variable in the stabilization of stress in nanocrystalline grains. The use of intrinsic segregation in alloys has already been shown to have a stabilizing influence on the grain size via segregation to grain boundaries (Section 2).

Fu et al. reported composition-dependent tunable residual stress in Fe-Pt alloy films [63]. When deposited as pure elements, Fe (the low mobility species) retained a tensile stress whereas $\mathrm{Pt}$ (the high mobility species) retained a compressive stress. In a mixed or alloy composition, the differences in mobility caused the higher mobility Pt to enrich the grain boundaries. Fig. 7 shows the residual stress in Fe-Pt alloy films as a function of composition. As the amount of Pt was increased (> $35 \% \mathrm{Pt})$, the films became more compressive and did not follow a linear rule of mixtures behavior. A wide range of stress, from $\sim 600 \mathrm{MPa}$ for a pure Fe film to - $1600 \mathrm{MPa}$ for a film with $\sim 21$ at. \% Fe, was observed as a function of composition. A near zero stress state is obtained in $\mathrm{Fe}_{0.65} \mathrm{Pt}_{0.35}$ film, marked by the arrow in Fig. 7.

Fig. 8 shows the atom maps and 1D composition profiles for $\mathrm{Fe}_{0.54} \mathrm{Pt}_{0.46}$ and $\mathrm{Fe}_{0.65} \mathrm{Pt}_{0.35}$ films, obtained from atom probe tomography. Even in an $\mathrm{Fe}$ rich film $\left(\mathrm{Fe}_{0.54} \mathrm{Pt}_{0.46}\right)$, $\mathrm{Pt}$ being a highly mobile atom and having a thermodynamic preference to enrich an Fe interface is enriched at the grain boundaries. This is visually observed in the atom map seen in Fig. 8 (a) and quantified in 1D composition profile in Fig. 8 (b) $[63,64]$. This is consistent with 
Chason's model of grain boundary diffusion. This film was reported to have a compressive stress value of $\sim-600 \mathrm{MPa}$. However, the $\mathrm{Fe}_{0.65} \mathrm{Pt}_{0.35}$ film was reported to have a near zero stress state. The atom map of $\mathrm{Fe}_{0.65} \mathrm{Pt}_{0.35}$ film, Fig. 8 (c), showed no visual enrichment of either Fe or Pt at the grain boundary as compared to the grain interior. This is quantified in Fig. 8 (d)'s 1D composition profile.

The results of the Fe-Pt alloy film clearly indicates that stress can be tuned by controlling the number of atoms flowing to the grain boundaries which can be macroscopically tuned by the bulk composition and the enthalpy of mixing and segregation. By increasing the amount of $\mathrm{Pt}$, a higher amount of $\mathrm{Pt}$ enriched the grain boundary making the film more compressive. Hence, the difference in the mobility of atoms resulted in a composition-dependent segregation of atoms to the grain boundaries and resulting stress state stabilization.

Using these two findings, Fu et al. modified Chason's equation for chemical potential difference which caused the flow of atoms to grain boundaries by adding $\mu_{c}$, which describes the change in chemical potential due to segregation. This is important because the chemical potential difference is the driving force for the flux of atoms from the surface to the grain boundary. The chemical potential difference between the surface and grain boundary, in the presence of a species that segregates to the grain boundary, would be given as:

$$
\Delta \mu^{\prime}=\mu_{s}-\mu_{g b}=\Delta \mu_{0}+\delta \mu_{s}+\sigma \Omega-\mu_{c}
$$

where $\Delta \mu_{0}=$ difference between the surface and grain boundary chemical potential in absence of growth, $\delta \mu_{s}=$ increase in surface chemical potential 
because of the flux of deposited atoms, $\sigma=$ stress along the grain boundary, $\Omega$ is the atomic volume, and $\mu_{c}=$ chemical potential rise in the grain boundary because of the segregation of chemically distinct, high mobility adatoms.

To the best of our knowledge, there are no other experimental results on the use of solutes to tune the stress states of a film. The use of such multispecies films, though more complex, has many advantages in elucidating the mechanisms associated with nanocrystalline stability in reference to stress generation. Similar to classic diffusion studies, where tracer atoms allow one to monitor the migration of species in a material, the use of different atoms here allows one to distinguish the chemical character of the nanocrystalline grain boundaries and its contribution to the stress evolution. Even more promising is the mixed mobility behavior between species providing the ability to tune the stress via solute enrichment of the boundaries. By controlling the grain boundary chemistry, a material dependent variable, rather than a processing dependent parameter, a potentially new means to regulate stresses in films is now available. Though these concepts appear simplistic, and film deposition is a complex and dynamical event, the promising results of section 2's thermodynamic stability maps (where stable segregation is predicted) and the experimental results of FePt [63] are proof-of-concepts where these two fields can be merged. The thermodynamic maps, developed in the nanocrystalline grain stabilization efforts of Section 2, can provide a design tool for thin films which could help tune the stress and reduce common film failures of delamination and cracking. 


\section{Conclusions and Future Challenges}

As the length scale of materials decreases to the nanometer regime, unique changes in material properties occur. One of the primary changes is the larger fraction of grain boundaries rendering them an important part of stability of nanocrystalline materials. Consequently, the ability to stabilize nanocrystalline grains is an area of active research. This review has provided a summary of highlights in both the nanocrystalline grain growth and nanocrystalline thin film stress evolution. Though each field can be considered distinct; recent developments in understanding the stabilizing impact of solute segregation to grain boundaries provides for an exciting opportunity to unify the two fields. In particular, the grain size stabilization efforts have shown the interrelationship of enthalpy of mixing and enthalpy of segregation to control nano-grain growth. An outcome of this work has been thermodynamic stability maps. At this time, only a handful of systems have been experimentally studied to test and verify these maps as a robust design tool. Direct evidence of solute segregation at the grain boundaries has only been obtained in a very few studies and more computational and experimental work is needed.

Using these thermodynamic maps, one can select systems where grain boundary enrichment could be used to regulate the stress in thin films, which commonly have nanometer grain sizes. Though thin film deposition is dynamic and some of the specific thermodynamic factors between the 'bulk' nanocrystalline stability and those in thin films will differ, the overarching ideas of solute segregation in either system provides the link between the two fields. The 
stability maps could provide guidance in identifying thin film systems where stress manipulation through segregation control is possible.

Recent experimental and modeling reports have provided a more clear understanding of how grain boundary area (e.g. grain size) contributes to the stress evolution of thin films. The migration of atoms into and out of grain boundaries has been proposed to control the stress and appears to be gaining traction as a valid model. A recent deposition of an alloy film, with one species enriching the boundaries, was shown to manipulate the stress from tensile to compression, with a zero-stress cross over. These results were understood using the grain boundary diffusion model. The zero-stress film showed no preference of segregation of either species to the boundary; this is suggestive that intrinsic material properties, such as thermodynamic segregation and mixing, will have a role in stress generation of thin films, analogous to the grain size stability against growth. To date, more work is still needed to firmly link these connections. Regardless of the current outstanding issues in either field, the promising results of atomic migration to nanocrystalline grains in regulating their stability appears to be a fruitful area of emerging research for both experiments and modeling.

\section{Acknowledgments:}

G.B. Thompson thanks Prof. M. O'Keefe of Missouri Science \& Technology for the invitation to write this review. M. Kapoor gratefully acknowledges the support from Sandia National Laboratories through the Department of Energy Office of Basic Energy Sciences and G.B. Thompson acknowledges support from the 
Army Research Office, grant W911NF1310436, Dr. Suveen Mathaudhu, program manager. 


\section{Captions}

1. Fig. 1: Volume fraction of intercrystalline regions, grain boundary, and triple junctions as a function of grain size in nano-crystalline Fe [1].

2. Fig. 2: Segregation energy as a function of bulk composition of $\mathrm{W}$ solute [10], (color available online).

3. Fig. 3 (a): Thermodynamic stability map showing three regions of stability for various combinations of solute and solvent. The solute is denoted by the element abbreviation and the solvent by the geometrical symbol [15] e.g. the $\mathrm{Mn}$ solute in the $\mathrm{Cu}$ solvent is the $\mathrm{Mn}$ abbreviation next to the filled blue circle in the pink region (labeled by the solid arrow); the $\mathrm{Au}$ solute in the Mo solvent is the Au abbreviation next to the open magenta circle in the green region (labeled by the dashed arrow). (b) Free energy surface with the minimum showing the stable grain size at the bulk solute concentration, at which this surface was calculated [12]. (color available online)

4. Fig. 4 (a): Normalized grain boundary energy calculated as a function of the grain boundary mole fraction of the solute, $\mathrm{Zr}$, for a series of grain sizes in Fe-Zr, and (b) Stability map for nanocrystalline Fe that plots solutes (stable - red) as a function of enthalpy of mixing and elastic enthalpy. The size of the dot is proportional to the amount of solute needed to stabilize a grain size of $25 \mathrm{~nm}$ [14], (color available online).

5. Fig. 5: (a) A one-micron Fe film sputter-deposited onto a Si wafer. As the film thickened, the intrinsic stress increased until it formed micro-cracks that relieved the stress and resulted in the film delaminating from the substrate. This represents an extreme example of how stress can result in catastrophic film failure. (b) Schematic cartoon of stress evolution during 
film deposition. The grey region shows the initial stage where isolated islands/clusters form and are under compression due to surface capillary effects. The blue region shows the stage where the islands coalesce and form grain boundaries. The pink region represents onset of postcoalescence stress generation. The black dots represent atoms within the grains and grey dots represent atoms at grain boundary. (color available online)

6. Fig. 6: Stress-thickness as a function of time for Cu films.

7. Fig.7: Residual stress as a function of composition, in a series of Fe-Pt alloy films. This illustrates the range of stress states as a function of composition with a zero stress state attained in $\mathrm{Fe}_{0.65} \mathrm{Pt}_{0.35}$ film [63], (color available online).

8. Fig.8: (a) $\mathrm{Pt}$ atom map of $\mathrm{Fe}_{0.54} \mathrm{Pt}_{0.46}$ alloy film, (b) $1 \mathrm{D}$ composition profile through the grain boundary, obtained from the cylindrical volume, showing Pt enrichment, (c) Pt atom map of $\mathrm{Fe}_{0.65} \mathrm{Pt}_{0.35}$ alloy film, and (d) 1D composition profile throughout the sample, obtained from the cylindrical volume, showing no preferential segregation [63], (color available online). 


\section{References}

[1]. G. Palumbo, S. J. Thorpe, et al. On the contribution of triple junctions to the structure and properties of nanocrystalline materials. Scripta Metallurgica et Materialia 24 (1990) 1347-1350.

[2]. G. B. Thompson, R. Banerjee, et al. Phase stability of bcc $\mathrm{Zr}$ in Nb/Zr thin film multilayers. Acta Materialia 51 (2003) 5285-5294.

[3]. T. Chookajorn, C. A. Schuh. Nanoscale segregation behavior and hightemperature stability of nanocrystalline W-20at.\% Ti. Acta Materialia 73 (2014) 128-138.

[4]. T. J. Rupert, W. Cai, et al. Abrasive wear response of nanocrystalline Ni-W alloys across the Hall-Petchbreakdown. Wear 298-299 (2013) 120-126.

[5]. R. Koch. The intrinsic stress of polycrystalline and epitaxial metal thin films. Journal of Physics: Condensed Matter 6 (1994) 9519-9550.

[6]. C. C. Koch, R. O. Scattergood, et al. High temperature stabilization of nanocrystalline grain size: Thermodynamic versus kinetic strategies. Journal of Materials Research 28 (2013) 1785-1791.

[7]. A. Detor, C. A. Schuh. Tailoring and patterning the grain size of nanocrystalline alloys. Acta Materialia 55 (2007) 371-379.

[8]. B. Farber, E. Cadel, et al. Phosphorus segregation in nanocrystalline Ni-3.6

at. \% $\mathrm{P}$ alloy investigated with the tomographic atom probe (TAP). Acta Materialia 48 (2000) 789-796. 
[9]. A. Detor, C. A. Schuh. Microstructural evolution during the heat treatment of nanocrystalline alloys. Journal of Materials Research 22 (2007) 3233-3248.

[10]. A. Detor, C. A. Schuh. Grain boundary segregation, chemical ordering and stability of nanocrystalline alloys: Atomistic computer simulations in the $\mathrm{Ni}-\mathrm{W}$ system. Acta Materialia 55 (2007) 4221-4232.

[11]. K. A. Darling, B. K. VanLeeuwen, et al. Thermal stability of nanocrystalline Fe-Zr alloys. Materials Science and Engineering: A 527 (2010) 3572-3580.

[12]. H. A. Murdoch, C. A. Schuh. Stability of binary nanocrystalline alloys against grain growth and phase separation. Acta Materialia 61 (2013) 21212132.

[13]. K. A. Darling, B. K. VanLeeuwen, et al. Stabilized nanocrystalline ironbased alloys: Guiding efforts in alloy selection. Materials Science and Engineering: A 528 (2011) 4365-4371.

[14]. K. A. Darling, M. A. Tschopp, et al. Mitigating grain growth in binary nanocrystalline alloys through solute selection based on thermodynamic stability maps. Computational Materials Science 84 (2014) 255-266.

[15]. H. A. Murdoch, C. A. Schuh. Estimation of grain boundary segregation enthalpy and its role in stable nanocrystalline alloy design. Journal of Materials Research 28 (2013) 2154-2163.

[16]. T. Chookajorn, H. A. Murdoch, et al. Design of stable nanocrystalline alloys. Science 337 (2012) 951-4. 
[17]. J. Leib, R. Mönig, et al. Direct Evidence for effects of grain structure on reversible compressive deposition stresses in polycrystalline gold films. Physical Review Letters 102 (2009) 256101-4.

[18]. C.-W. Pao, S. Foiles, et al. Thin film compressive stresses due to adatom insertion into grain boundaries. Physical Review Letters 99 (2007) 36102-1-4.

[19]. D. Flötotto, Z. M. Wang, et al. Effect of adatom surface diffusivity on microstructure and intrinsic stress evolutions during Ag film growth. Journal of Applied Physics 112 (2012) 043503-1-9.

[20]. E. Chason, B. Sheldon, et al. Origin of compressive residual stress in polycrystalline thin films. Physical Review Letters 88 (2002) 156103-1-4.

[21]. J. W. Shin, E. Chason. Compressive stress generation in Sn thin films and the role of grain boundary diffusion. Physical Review Letters 103 (2009) 056102$1-4$.

[22]. E. Chason, J. W. Shin, et al. Kinetic model for dependence of thin film stress on growth rate, temperature, and microstructure. Journal of Applied Physics 111 (2012) 083520-1-10.

[23]. D. Magnfält, G. Abadias, et al. Atom insertion into grain boundaries and stress generation in physically vapor deposited films. Applied Physics Letters 103 (2013) 051910-1-4.

[24]. C.-W. Pao, D. Srolovitz, et al. Effects of surface defects on surface stress of $\mathrm{Cu}(001)$ and $\mathrm{Cu}(111)$. Physical Review B 74 (2006) 155437-1-8. 
[25]. M. J. Buehler, A. Hartmaier, et al. Atomistic and continuum studies of cracklike diffusion wedges and associated dislocation mechanisms in thin films on substrates. Journal of the Mechanics and Physics of Solids 51 (2003) 21052125.

[26]. C.-W. Pao, D. Srolovitz. Stress and morphology evolution during island growth. Physical Review Letters 96 (2006) 186103-1-4.

[27]. S. J. Hearne, J. A. Floro. Mechanisms inducing compressive stress during electrodeposition of Ni. Journal of Applied Physics 97 (2005) 014901-1-6.

[28]. C. C. Koch, R. O. Scattergood, et al. Stabilization of nanocrystalline grain sizes by solute additions. Journal of Materials Science 43 (2008) 7264-7272.

[29]. E. Nes, N. Ryum, et al. On the Zener drag. Acta Metallurgica 33 (1985) 1122.

[30]. J. W. Cahn. The impurity-drag effect in grain boundary motion. Acta Metallurgica 10 (1962) 789-798.

[31]. J. Weissmuller. Alloy effects in nanostructures. Nanostructured Materials 3 (1993) 261-272.

[32]. F. Liu, R. Kirchheim. Nano-scale grain growth inhibited by reducing grain boundary energy through solute segregation. Journal of Crystal Growth 264 (2004) 385-391.

[33]. J. Weissmuller. Some basic notions on nanostructured solids. Materials Science and Engineering: A 179-180 (1994) 102-107. 
[34]. J. Weissmuller, P. Bunzel, et al. Two phase equilibrium in small alloy particles. Journal of Materials Research 9 (1994) 4-7.

[35]. T. Hentschel, D. Isheim, et al. Nanocrystalline Ni-3.6 at. \% P and its transformation sequence studied by atom-probe field ion microscopy. Acta Materialia 48 (2000) 933-941.

[36]. R. Kirchheim. Grain coarsening inhibited by solute segregation. Acta Materialia 50 (2002) 413-419.

[37]. F. Liu, R. Kirchheim. Grain boundary saturation and grain growth. Scripta Materialia 51 (2004) 521-525.

[38]. J. Trelewicz, C. A. Schuh. Grain boundary segregation and thermodynamically stable binary nanocrystalline alloys. Physical Review B 79 (2009) 94112-1-13.

[39]. K. A. Darling, R. N. Chan, et al. Grain-size stabilization in nanocrystalline FeZr alloys. Scripta Materialia 59 (2008) 530-533.

[40]. M. Saber, H. Kotan, et al. Thermodynamic stabilization of nanocrystalline binary alloys. Journal of Applied Physics 113 (2013) 063515-1-10.

[41]. P. C. Millett, R. P. Selvam, et al. Stabilizing nanocrystalline materials with dopants. Acta Materialia 55 (2007) 2329-2336.

[42]. M. A. Atwater, K. A. Darling, A Visual Library of Stability in Binary Metallic Systems:The Stabilisation of Nanocrystalline Grain Size by Solute Addition: Part 1 (No. ARL-TR-6007), 2012, DTIC\#ADA561871. 
[43]. T. Chookajorn, C. A. Schuh. Thermodynamics of stable nanocrystalline alloys: A Monte Carlo analysis. Physical Review B 89 (2014) 64102-1-10.

[44]. R. Koch, D. Hu, et al. Compressive stress in polycrystalline Volmer-Weber films. Physical Review Letters 94 (2005) 146101-1-4.

[45]. J. Barbosa, B. Almeida, et al. Stress induced magnetic anisotropy on $\mathrm{BaTiO}-\mathrm{CoFe} 2 \mathrm{O} 4$ nanogranular composite thin films. Journal of Non-Crystalline Solids 354 (2008) 5250-5252.

[46]. A. Miyamura, K. Kaneda, et al. Effects of internal stress on photocatalytic properties of TiO2 films. Thin Solid Films 516 (2008) 4603-4608.

[47]. J. Zhu, Y. Li, et al. Effects of compressive and tensile stress on the growth mode of epitaxial oxide films. Ceramics International 34 (2008) 967-970.

[48]. C. T. Chuang, C. K. Chao, et al. Effects of internal stresses on the mechanical properties of deposition thin films. Journal of Materials Processing Technology 201 (2008) 770-774.

[49]. A. Tiwari, M. Senthil Kumar. Effect of addition of $\mathrm{Ni}$ on the structure and giant magnetoresistance in Fe-Cu films. Physica B: Condensed Matter $\mathbf{3 8 7}$ (2007) 63-68.

[50]. I. Mizushima, P. T. Tang, et al. Residual stress in Ni-W electrodeposits. electrochimica acta 51 (2006) 6128-6134.

[51]. J. Matovic. Application of Ni electroplating techniques towards stress-free microelectromechanical system-based sensors and actuators. Proceedings of 
the Institution of Mechanical Engineers, Part C: Journal of Mechanical Engineering Science 220 (2006) 1645-1654.

[52]. R. Abermann, R. Koch. The internal stress in thin silver, copper and gold films. Thin Solid Films 129 (1985) 71-78.

[53]. R. C. Cammarata, T. M. Trimble, et al. Surface stress model for intrinsic stresses in thin films. Journal of Materials Research 15 (2000) 2468-2474.

[54]. W. D. Nix, B. M. Clemens. Crystallite coalescence- A mechanism for intrinsic tensile stresses in thin films. Journal of Materials Research 14 (1999) 3467-3473.

[55]. R. W. Hoffman. Stresses in thin films: The relevance of grain boundaries and impurities. Thin Solid Films 34 (1976) 185-190.

[56]. L. B. Freund, E. Chason. Model for stress generated upon contact of neighboring islands on the surface of a substrate. Journal of Applied Physics 89 (2001) 4866-73.

[57]. G. Thurner, R. Abermann. Internal stress and structure of ultrahigh vacuum evaporated $\mathrm{Cr}$ and Fe films and their dependence on substrate temperature and oxygen partial pressure during deposition. Thin Solid Films 192 (1990) 277-285.

[58]. M. Pletea, R. Koch, et al. In situ stress evolution during and after sputter deposition of Al thin films. Journal of Physics: Condensed Matter 21 (2009) 225008-1-8.

[59]. C. Friesen, S. C. Seel, et al. Reversible stress changes at all stages of Volmer-Weber film growth. Journal of Applied Physics 95 (2004) 1011-20. 
[60]. C. Friesen, C. V. Thompson. Comment on "Compressive Stress in Polycrystalline Volmer-Weber Films". Physical Review Letters 95 (2005).

[61]. A. L. Shull, F. Spaepen. Measurements of stress during vapor deposition of copper and silver thin films and multilayers. Journal of Applied Physics 80 (1996) 6243-56.

[62]. G. Abadias, A. Fillon, et al. Real-time stress evolution during early growth stages of sputter-deposited metal films: Influence of adatom mobility. Vacuum 100 (2014) 36-40.

[63]. B. Fu, G. B. Thompson. Compositional dependent thin film stress states. Journal of Applied Physics 108 (2010) 043506-1-6.

[64]. B. Yang, M. Asta, et al. Equilibrium Monte Carlo simulations of A1-L10 ordering in FePt nanoparticles. Scripta Materialia 53 (2005) 417-422. 


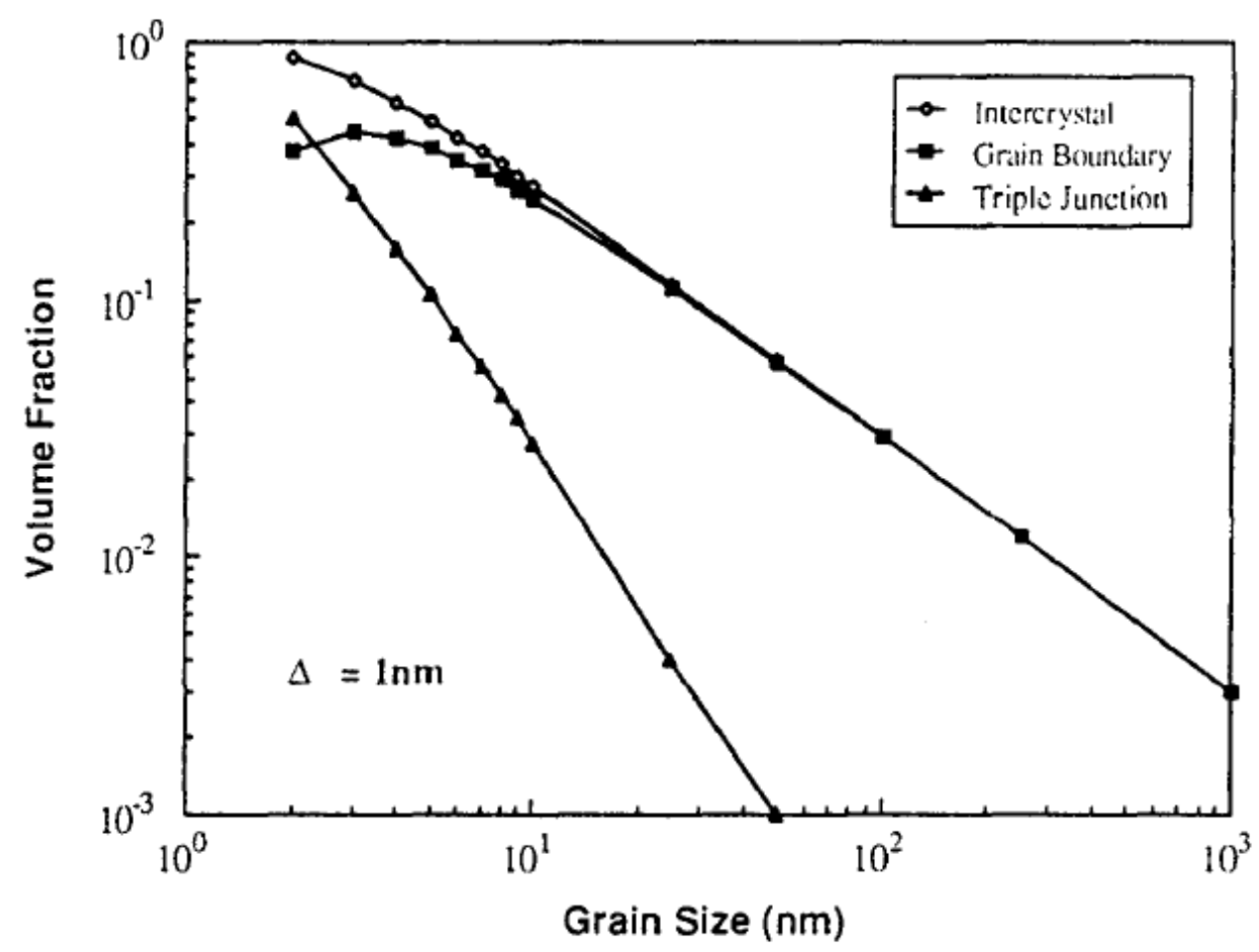

Fig. 1: Volume fraction of intercrystalline regions, grain boundary, and triple junctions as a function of grain size in nano-crystalline Fe [1]. 


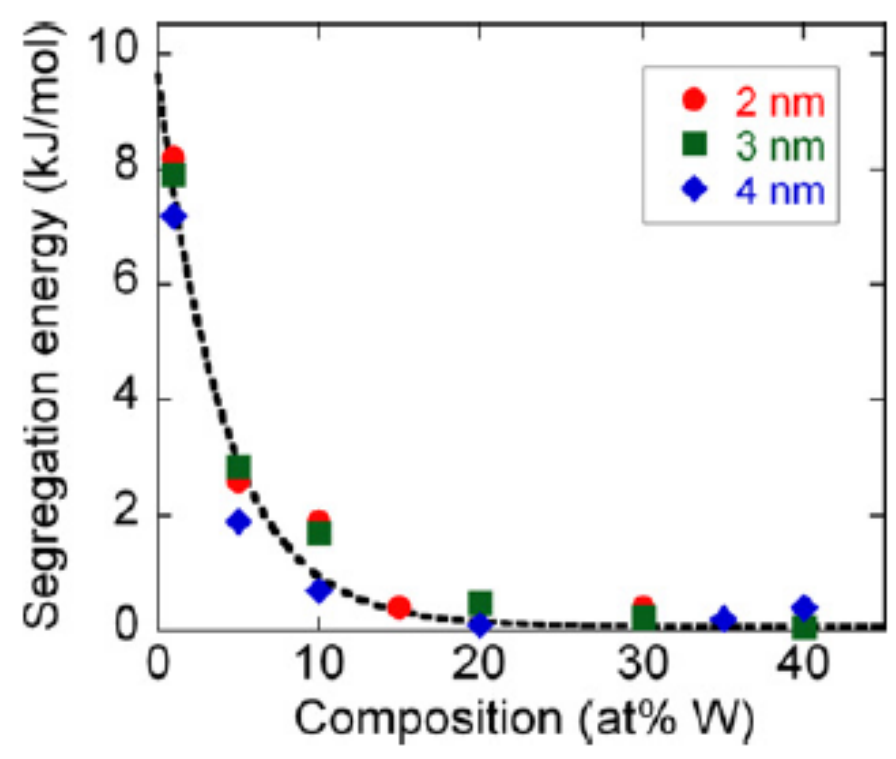

Fig. 2: Segregation energy as a function of bulk composition of W solute [10]. (color available online) 
(a)

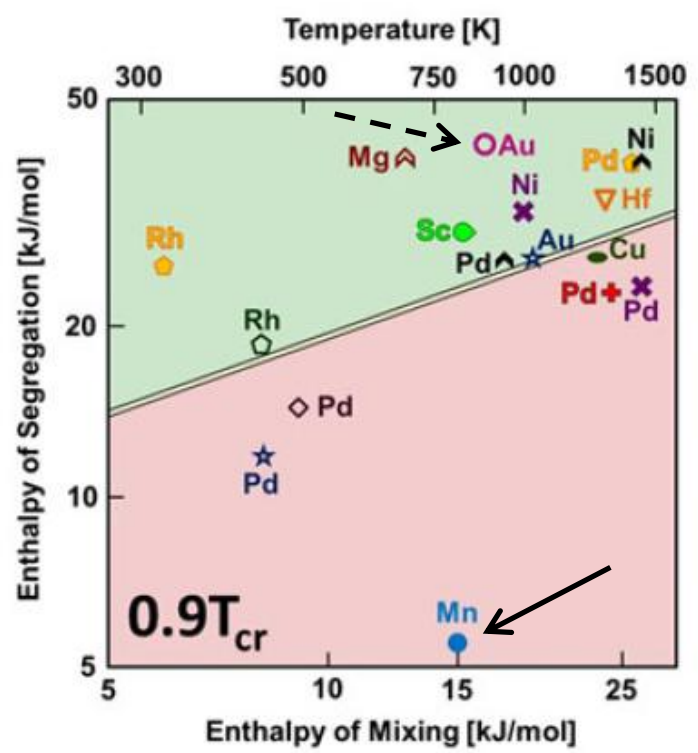

(b)

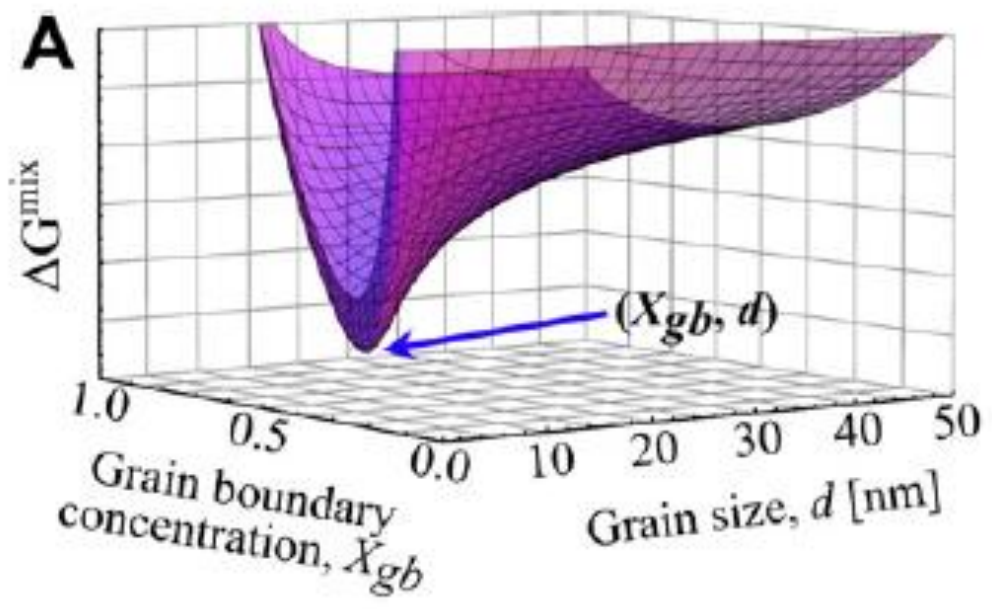

Fig. 3 (a): Thermodynamic stability map showing three regions of stability for various combinations of solute and solvent. The solute is denoted by the element abbreviation and the solvent by the geometrical symbol [15] - e.g. the Mn solute in the Cu solvent is the $\mathrm{Mn}$ abbreviation next to the filled blue circle in the pink region (labeled by the solid arrow); the Au solute in the Mo solvent is the Au abbreviation next to the open magenta circle in the green region (labeled by the dashed arrow). (b) Free energy surface with the minimum showing the stable grain size at the bulk solute concentration, at which this surface was calculated [12]. (color available online) 

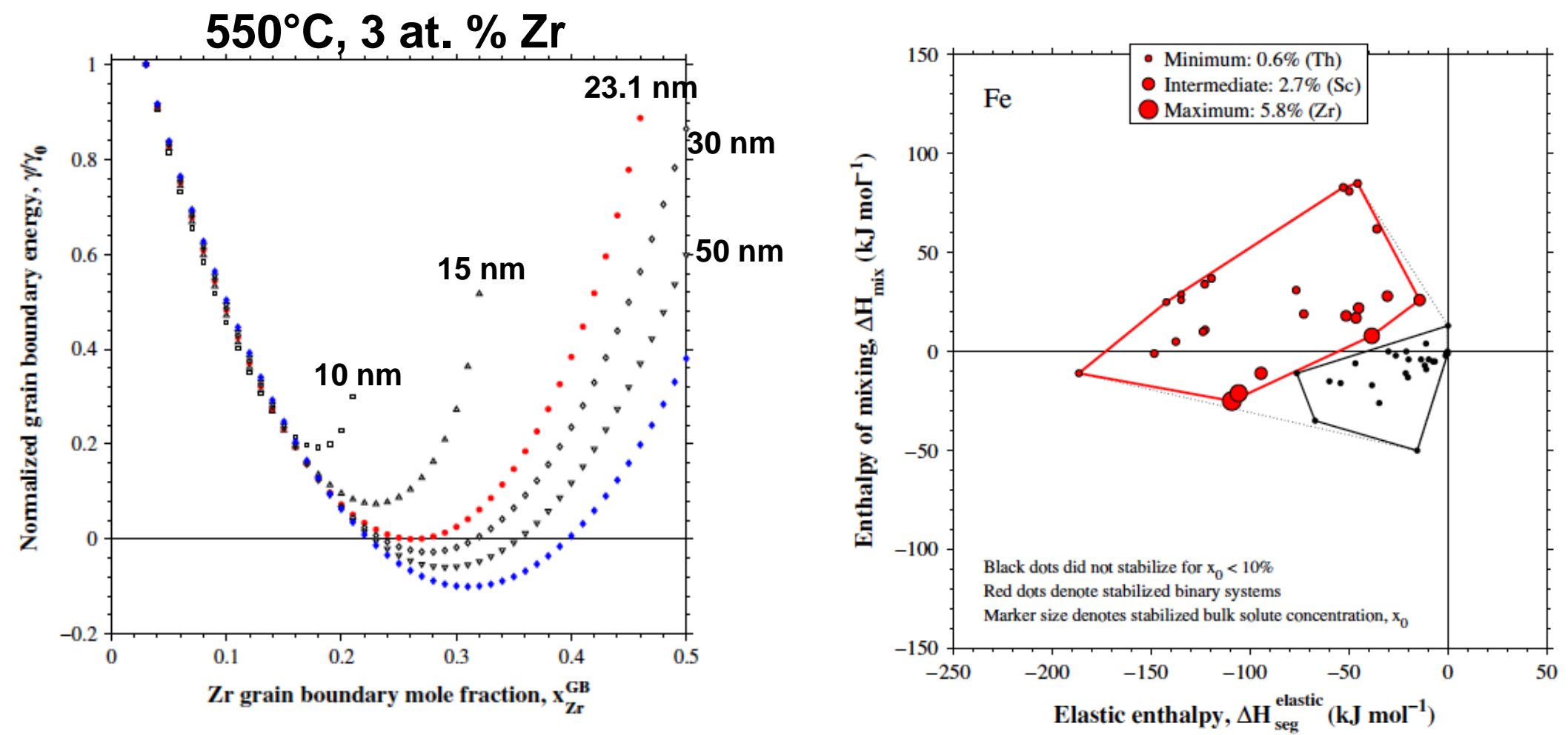

Fig. 4 (a): Normalised grain boundary energy calculated as a function of the grain boundary mole fraction of the solute, $\mathrm{Zr}$, for a series of grain sizes in Fe-Zr, and (b) Stability map for nanocrystalline Fe that plots solutes (stable - red) as a function of enthalpy of mixing and elastic enthalpy. The size of the dot is proportional to the amount of solute needed to stabilise a grain size of $25 \mathrm{~nm}$ [14]. (color available on-line) 


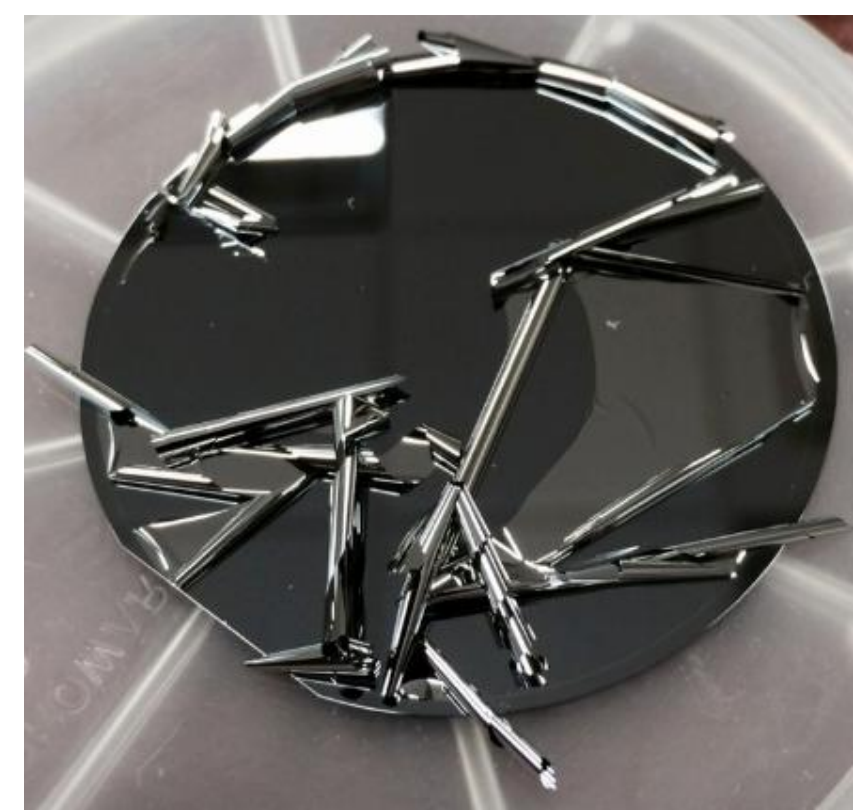

(a)

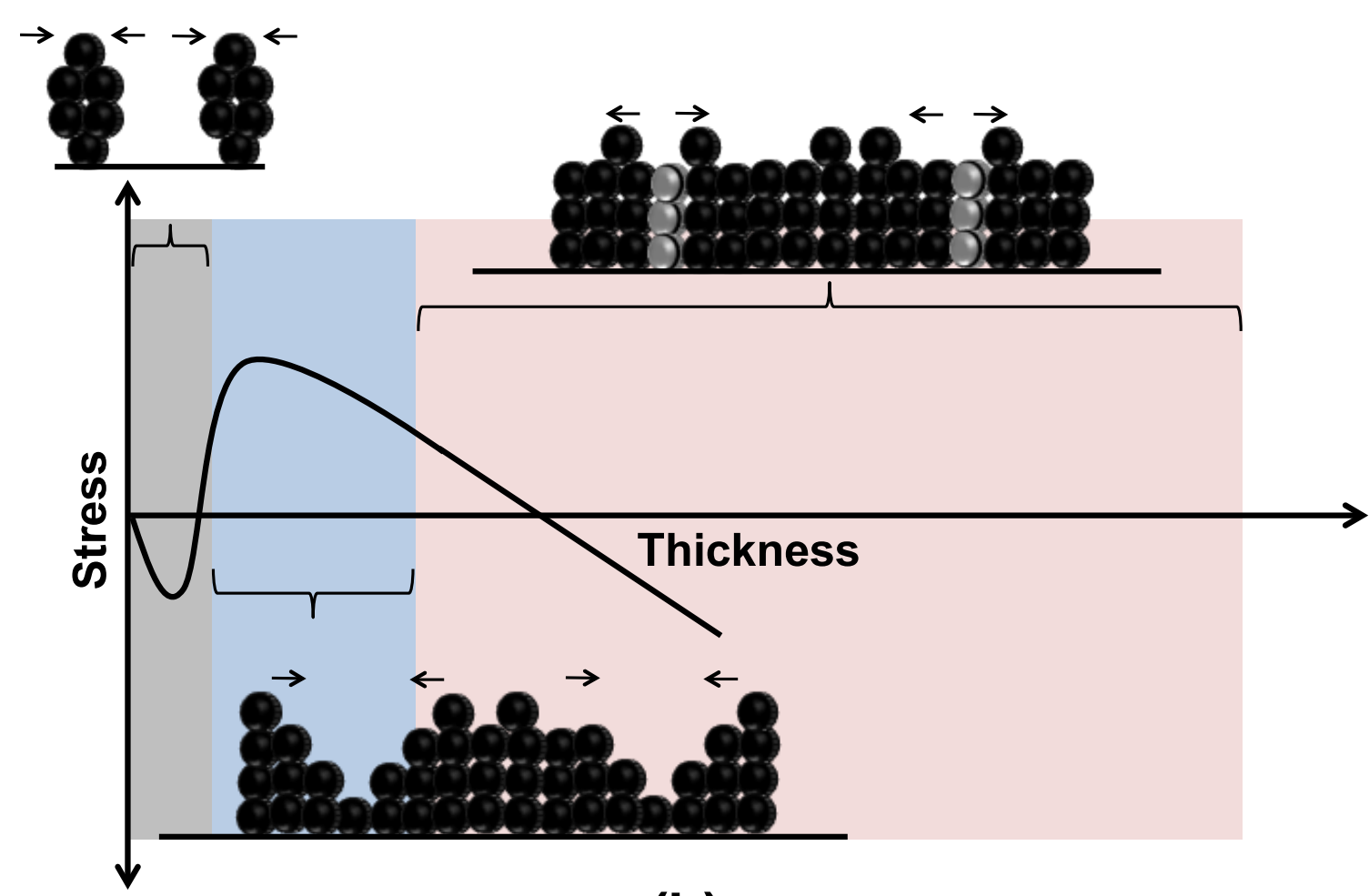

(b)

Fig. 5: (a) A one-micron Fe film sputter-deposited onto a Si wafer. As the film thickened, the intrinsic stress increased until it formed micro-cracks that relieved the stress and resulted in the film delaminating from the substrate. This represents an extreme example of how stress can result in catastrophic film failure. (b) Schematic cartoon of stress evolution during film deposition. The grey region shows the initial stage where isolated islands/clusters form and are under compression due to surface capillary effects. The blue region shows the stage where the islands coalesce and form grain boundaries. The pink region represents onset of post-coalescence stress generation. The black dots represent atoms within the grains and grey dots represent atoms at grain boundary. (color available online) 


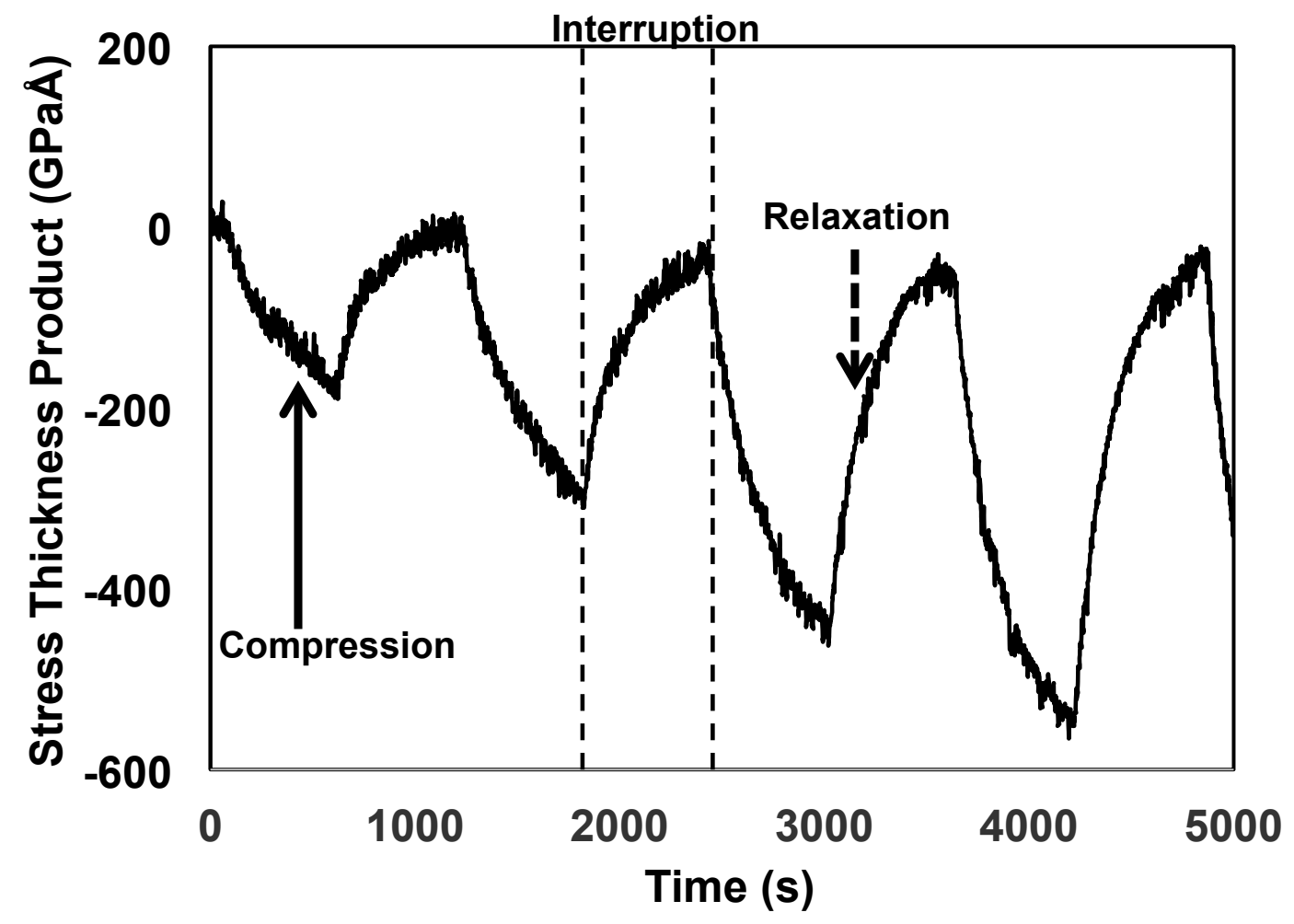

Fig. 6: Stress-thickness as a function of time for Cu films. 


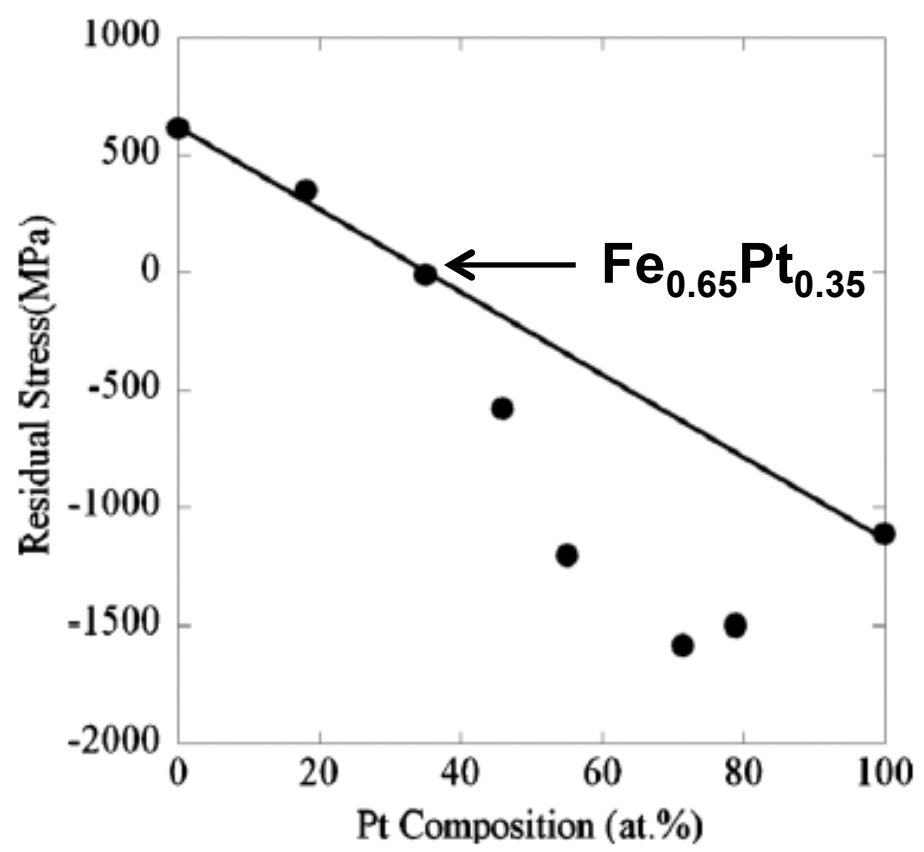

Fig.7: Residual stress as a function of composition, in a series of Fe-Pt alloy films. This illustrates the range of stress states as a function of composition with a zero stress state attained in $\mathrm{Fe}_{0.65} \mathrm{Pt}_{0.35}$ film [63]. 
(a)

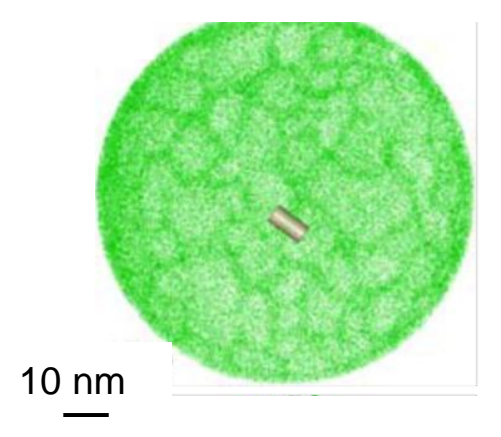

(c)

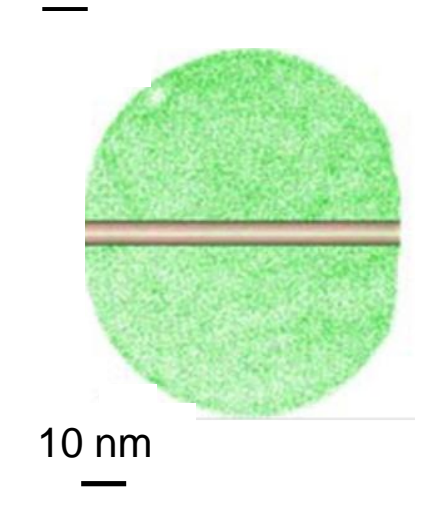

(b)

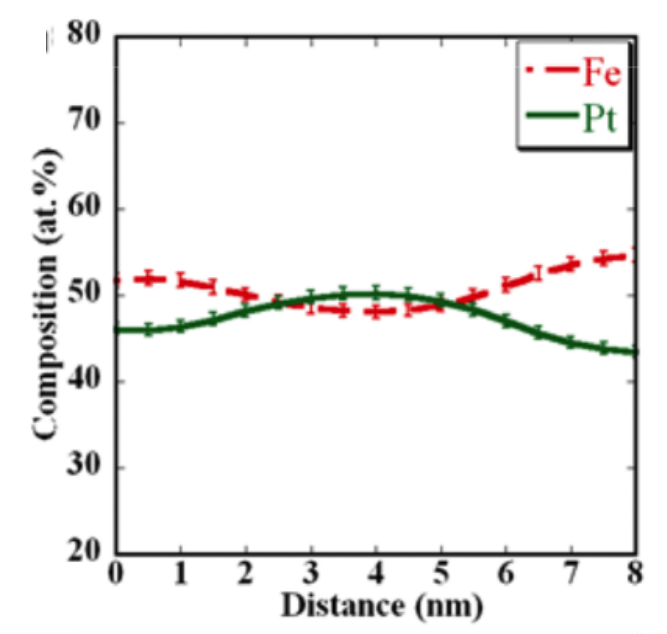

(d)

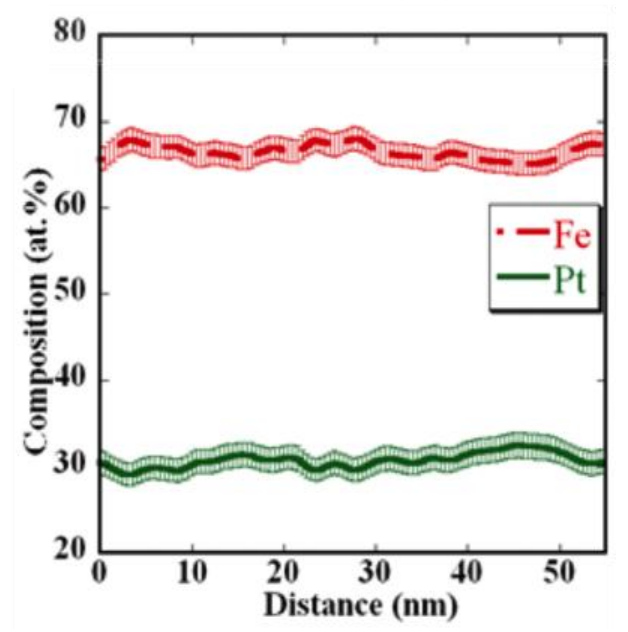

Fig.8: (a) Pt atom map of $\mathrm{Fe}_{0.54} \mathrm{Pt}_{0.46}$ alloy film, (b) $1 \mathrm{D}$ composition profile through the grain boundary, obtained from the cylindrical volume, showing Pt enrichment, (c) Pt atom map of $\mathrm{Fe}_{0.65} \mathrm{Pt}_{0.35}$ alloy film, and (d) $1 \mathrm{D}$ composition profile throughout the sample, obtained from the cylindrical volume, showing no preferential segregation [63] . The cylindrical volume shown in atom maps in (a) and (c) represents the volume through which the 1D composition profile was obtained. (color available online) 